A Bayesian Inference Approach to the Development of a Multidirectional Pedestrian Stream Model

Siqi Xie ${ }^{1}$ and S.C. Wong ${ }^{2}$

Department of Civil Engineering, The University of Hong Kong, Hong Kong

${ }^{1}$ Department of Civil Engineering, The University of Hong Kong, Pokfulam Road, Hong Kong, China (Corresponding Author). E-mail: seakay@hku.hk; Tel.: +852-2859-2662; Fax: +852-2517-0124

${ }^{2}$ Department of Civil Engineering, The University of Hong Kong, Pokfulam Road, Hong Kong, China. E-mail: hhecwsc@hku.hk; Tel.: +852-2859-1964; Fax: +852-2559-5337 


\title{
A Bayesian Inference Approach to the Development of a Multidirectional Pedestrian Stream Model
}

\author{
Siqi Xie and S.C. Wong \\ Department of Civil Engineering, The University of Hong Kong, Pokfulam Road, Hong Kong \\ E-mail: seakay@hku.hk
}

\begin{abstract}
In this paper, we develop a mathematical model to represent the conflicting effects of multidirectional pedestrian flows in a large crowd. The model is formulated based on Drake's model of traffic flow. Rather than relate the speed of a pedestrian stream solely to the pedestrian density, we introduce the flow ratio and intersecting angle between streams as variables. To calibrate the model, data collection was conducted through the video recording of pedestrian movements on a pedestrian street in Mong Kok, Hong Kong. Bayesian inference was adopted to calibrate the parameters based on the information from a previous experiment. Finally, we study the relationships among the speed, density, flow and intersecting angles of the pedestrian streams and predict how these variables affect the pedestrian movements.
\end{abstract}

Keywords: Pedestrian; Multidirectional flow; Measurement; Empirical studies; Bayesian inference

\section{INTRODUCTION}

Walking is an environmentally friendly mode of transportation. A good understanding of pedestrian activities and effective planning of walking facilities are particularly important for densely populated Asian cities such as Hong Kong. Previous studies have used observational surveys and controlled experiments to examine unidirectional and bidirectional pedestrian streams. Video recording has been widely applied as a survey method in these studies, as it is economic, convenient and has relatively high accuracy. The video recordings provide a real-time record of pedestrian movements from which the position of each individual pedestrian at any moment can be extracted. Although multidirectional pedestrian streams are common in daily life, few previous studies have modeled multidirectional streams. Hence, in this study, we video recorded the pedestrian movements at a crosswalk on a pedestrian street in Mong Kok and extracted the relevant data to develop a mathematical model that reflects the relationships among specific macroscopic quantities related to the multidirectional pedestrian flow, including speed, density, flow and the intersecting angle between conflicting streams.

The continuum approach has been widely applied in macroscopic pedestrian flow modeling. Hughes $(2000 ; 2002)$ proposed the continuum theory for two-dimensional pedestrian flows in which the pedestrians are regarded as the 'continuum fluid' and gave equations describing single and multiple types of pedestrians. Based on Hughes' (2000) single pedestrian type model, Jiang et al. (2009) proposed a reactive dynamic continuum-user equilibrium model to simulate bidirectional pedestrian flows. Huang et al. (2009) revisited the model for multiple pedestrian types and further developed an efficient solution algorithm. Jiang et al. (2012) extended the study to the simulation of pedestrian counter-flow. In addition, Xiong et al. (2011) proposed a high-order computational scheme for the Jiang et al. (2009) model, which proved to be more efficient than the first-order method. Xia et al. (2009) also developed a dynamic continuum pedestrian flow model taking the pedestrians' memories of the shortest route into consideration. Although these studies involved little empirical data, they examined different types of bidirectional pedestrian flows, including counter-flows and crossing movements with certain intersecting angles, which provide very useful information for subsequent studies based on empirical data.

There are few studies on multidirectional pedestrian flows in the literature. Blue et al. (2000) and Abdelghany et al. (2005) adopt cellular automata micro-simulation. Asano et al. (2007) proposed the cell 
transmission model for multidirectional pedestrian flow, in which a converted density is used to take the conflict between pedestrians into account. Given the limited research on the continuum modeling of multidirectional pedestrian flows, it is worth examining some of the related empirical studies on bidirectional pedestrian flows, as multidirectional pedestrian flows can be regarded as combinations of multiple pairs of bidirectional flows.

The counter flow is the most common form of bidirectional pedestrian flow. Daamen and Hoogendoorn (2003), Hoogendoorn and Daamen (2005) and Kretz et al. (2006a) performed experiments for pedestrian counter flows in corridors with different widths. Kretz et al. (2006a) found that the performance of the pedestrian flow, in terms of macroscopic quantities such as speed, flux and the passing of time, was not necessarily lower than that for the situation without counter flow, as the pedestrians were able to increase the efficiency of their use of space to a certain degree and therefore compensate for the existence of the counter flow. In addition, Kretz et al. (2006b) and Helbing et al. (2005) observed self-organizational phenomena in a series of controlled experiments on bidirectional pedestrian flows in bottlenecks with different widths. The investigation by Ando et al. (1988) is one of the few studies on bidirectional pedestrian flows to include an oblique intersecting angle. The consideration of intersecting angle is useful for modeling pedestrian movements in some crossing facilities, such as scramble-phase signalized intersections (Hediyeh et al., 2014a). Wong et al. (2010) also performed controlled experiments where pedestrians were asked to walk in designed directions at an oblique angle of $45^{\circ}, 90^{\circ}, 135^{\circ}$ and $180^{\circ}$, and they found the conflicting effect maximizes when the two streams form an $180^{\circ}$ intersecting angle. However, Zhang and Seyfried (2014) reported that they found no apparent difference with respect to the intersecting angle $90^{\circ}$ and $180^{\circ}$ based on their experiments, although they did not perform experiments on the situations with other intersecting angles.

Besides experimental studies, observational surveys are also important in model development for bidirectional pedestrian flows. Lam et al. (2002) conducted surveys on bidirectional pedestrian flows at a signalized crosswalk in Hong Kong. They examined the relationship between speed and flow under different conditions, including crosswalks with or without LRT stations. They also investigated the effect of bidirectional flow by incorporating the ratio between the flow of one stream and that of the total stream into the model. In addition, Lam et al. (2003) conducted surveys at indoor walkways in commercial and retail districts in Hong Kong. Similar to their previous study, they adopted the bidirectional pedestrian flow ratio and formulated a generalized walking time function that included the flow ratio and was calibrated with the data from the field surveys. This finding indicates that the speed of a pedestrian stream is not only dependent on the flow density but also the flow rate of the stream. Lam and Cheung (2000) also summarized the data from their observational surveys at MTR stations, signalized crosswalks and indoor and outdoor walkways, which provide useful information for identifying the characteristics of pedestrian flows in Hong Kong. Hediyeh et al. (2014b) studied the spatio-temporal gait parameters of pedestrian walking behavior using computer vision techniques in downtown Vancouver, Canada.

While controlled experiments can cover the full range of model parameters and conditions (Wong et al., 2010) and produce reliable modeling results, observational surveys represent the real situation and the data from these surveys can be used to verify the results obtained in the controlled experiments. Moussaid et al. (2009) provided a good example of how to move from controlled experiments to field observations. They first quantified the interaction effects in a social force model by performing a series of experiments, and then collected observational data to compare with the predictions from the model.

As Wong et al. (2010) conducted controlled experiments on bidirectional pedestrian streams with intersecting angles of $0,45,90,135$ and 180 degrees with different levels of density. Accordingly, their experimental data can provide reliable information on the full range of densities and intersecting angles. Xie et al. (2013) also adopted the Bayesian method to develop an improved bidirectional pedestrian stream model, based on the prior distribution provided from the data from the controlled experiment investigated in this paper and calibrated with observational data collected from a real crosswalk. In this 
paper, we propose a mathematical model of n-directional pedestrian flows. The Bayesian method is then used to calibrate the model based on the prior distribution from the results of the controlled experiments (Wong et al., 2010) and observational surveys (Xie et al., 2013). A new set of observational data was also collected from a crosswalk on a pedestrian street in Mong Kok, Hong Kong. This set of field data is used to calibrate the model parameters.

\section{DATA}

\subsection{Site Conditions}

The crosswalk between Nelson Street and Sai Yeung Choi Street South was selected for the observation of four-directional pedestrian flows. The crosswalk is located at the heart of a pedestrian street in Mong Kok. The pedestrian flows are particularly dense during weekends. Therefore, the video recording was conducted on a Sunday afternoon, when many people come to the area to shop. The region of interest (ROI) is the area within the $12.376 \mathrm{~m}$ long and $4.31 \mathrm{~m}$ wide rectangle depicted in Figure 1. The four $3 \mathrm{~m}$ x $3 \mathrm{~m}$ subareas in the middle of the ROI (Figure 1) were selected for data processing. The camera was installed in a building next to the site at $7.02 \mathrm{~m}$ above ground level. The points $\mathrm{A}, \mathrm{B}, \mathrm{C}$ and $\mathrm{D}$ that define the ROI served as control points for the camera calibration.

[Insert Figure 1 Here]

\subsection{Classification of Directions}

Although the directions of the pedestrian streams were specified in the controlled experiment, the pedestrian movements in the field observation first have to be carefully defined. As video recording was adopted for the observation and the paths of the pedestrians' movements were extracted for analysis, we can easily identify the origins and destinations of the pedestrians in the study area. As it is commonly accepted to consider the flow from all origins to a particular destination as a copy of the flow in multiple OD networks (Potts \& Oliver, 1972), we grouped the pedestrians walking toward the same destination as a copy of one flow, i.e. one stream of pedestrians. Considering the site conditions in Figure 1, the pedestrian movements in the field were classified according to the destinations (i.e. the edges of the crossing) that the pedestrians were walking toward.

[Insert Figure 2 Here]

\subsection{Summary of Data}

The data extracted from the observational survey are summarized in Table 1. Although the pedestrian flows were recorded continuously, the pedestrian movements were extracted in 2 s (i.e., 10 frames) data blocks. In total, 22,632 trajectories were extracted. As shown in Table 1, the average speed for the field observation is $0.96 \mathrm{~m} / \mathrm{s}$ and the average density is $0.65 \mathrm{ped} / \mathrm{m}^{2}$.

[Insert Table 1 Here]

The average speed and density of each of the four streams shown in Figure 2 are summarized in Table 2.

[Insert Table 2 Here] 
As shown in Table 2, the average densities of Streams 1 and 3 are 0.222 and $0.263 \mathrm{ped} / \mathrm{m}^{2}$, respectively, while the respective values for Streams 2 and 4 are 0.079 and $0.084 \mathrm{ped} / \mathrm{m}^{2}$. This indicates that the majority of pedestrians (about 75\%) walk along Sai Yeung Choi Street South (Streams 1 and 3) while fewer pedestrians cross the junction with Nelson Street (Streams 2 and 4). However, the average speeds of the four streams are close, ranging from $0.92 \mathrm{~m} / \mathrm{s}$ to $1.00 \mathrm{~m} / \mathrm{s}$, which reveals that the density difference between streams does not significantly affect their speed. Therefore, factors other than the density should be taken into account in the model formulation.

\section{MODEL DEVELOPMENT}

\subsection{Proposed Model}

Similar to the bidirectional pedestrian stream model proposed by Xie et al. (2013), the model proposed for multidirectional pedestrian flow is modified based on Drake's one-dimensional traffic model (1967) as Eq.(1). The flow ratio is introduced to represent the movement of each stream. A coefficient $\alpha$ is also added to provide flexibility for the angle term. The model also assumes that the conflicting effects of the multidirectional flows are accumulated as the product of that from each conflicting stream.

$$
V_{i}=V_{f} \exp \left(-\theta \rho_{t}^{2}\right) \prod_{j=1}^{n} \exp \left[-\beta\left(1-\frac{V_{i} \rho_{i}}{V_{i} \rho_{i}+V_{j} \rho_{j}}\right)\left(1-\cos \alpha \varphi_{i j}\right)\left(\rho_{i}+\rho_{j}\right)\right]
$$

where

$\mathrm{V}_{\mathrm{i}}$ is the speed of the $\mathrm{i}^{\text {th }}$ stream;

$\mathrm{V}_{\mathrm{f}}$ is the free-flow speed;

$\rho_{t}$ is the total density of all streams;

$\rho_{\mathrm{i}}$ is the density of the $\mathrm{i}^{\text {th }}$ stream;

$\varphi_{\mathrm{ij}}$ is the intersecting angle between the $\mathrm{i}^{\text {th }}$ and $\mathrm{j}^{\text {th }}$ stream (if $\mathrm{j}=\mathrm{i}$, then $\varphi_{\mathrm{ij}}=0$ ), which is estimated based on the pedestrians' walking paths of the two streams; and

$\theta, \beta$ and $\alpha$ are coefficients.

The model satisfies a series of boundary conditions:

1. When $\mathrm{j}=\mathrm{i}, \varphi_{\mathrm{ij}}=0$, there is no conflicting effect induced by the $\mathrm{i}^{\text {th }}$ stream.

2. The conflicting effect of the other stream of pedestrians should be symmetrical about $180^{\circ}$.

3. When the walking facility is nearly empty, the speed of any stream should approach the free-flow speed, i.e., $V_{i} \rightarrow V_{f}$ when $\rho_{t} \rightarrow 0$.

4. When the walking facility is nearly empty, the flow of any stream should approach zero, i.e., $q_{i} \rightarrow 0$ when $\rho_{t} \rightarrow 0$ because $q_{i}=V_{i} \rho_{i}$.

5. When the walking facility is nearly empty, the addition of a pedestrian to any stream of pedestrians does not affect the speed of the $\mathrm{i}^{\text {th }}$ stream, i.e., $\partial V_{i} / \partial \rho_{i} \rightarrow 0$ when $\rho_{t} \rightarrow 0$.

The model can be interpreted as the stream speed reduction induced by the isotropic effect and the conflicting effect. The first exponential term is similar to that in the Drake model and the model proposed by Xie et al. (2013) and represents the isotropic effect. The product of the sequence represents the accumulation of the conflicting effects from all the other streams. As the conflicting effect should depend on both the density and movement of the streams, the proposed model introduces the flow ratio (the flow of the $\mathrm{i}^{\text {th }}$ stream over the total flow of the $\mathrm{i}^{\text {th }}$ and $\mathrm{j}^{\text {th }}$ streams) to model the conflicting effect induced from 
the other streams. The intersecting angle between the $\mathrm{i}^{\text {th }}$ stream and each of the other streams is also considered as a factor that may influence the speed of the $i^{\text {th }}$ stream.

As the speed of one stream depends on the speed and density of each stream, as well as the intersecting angle between that stream and each of the other streams, in the completed form of the model, the corresponding $n$ streams should group together to form a set of structural equations. Therefore, the finalized model is as follows:

$$
\begin{aligned}
& V_{1}=V_{f} \exp \left(-\theta \rho_{t}^{2}\right) \prod_{j=1}^{n} \exp \left[-\beta\left(1-\frac{V_{1} \rho_{1}}{V_{1} \rho_{1}+V_{j} \rho_{j}}\right)\left(1-\cos \alpha \varphi_{1 j}\right)\left(\rho_{1}+\rho_{j}\right)\right] \\
& \ldots \\
& V_{i}=V_{f} \exp \left(-\theta \rho_{t}^{2}\right) \prod_{j=1}^{n} \exp \left[-\beta\left(1-\frac{V_{i} \rho_{i}}{V_{i} \rho_{i}+V_{j} \rho_{j}}\right)\left(1-\cos \alpha \varphi_{i j}\right)\left(\rho_{i}+\rho_{j}\right)\right] \\
& \ldots \\
& V_{n}=V_{f} \exp \left(-\theta \rho_{t}^{2}\right) \prod_{j=1}^{n} \exp \left[-\beta\left(1-\frac{V_{n} \rho_{n}}{V_{n} \rho_{n}+V_{j} \rho_{j}}\right)\left(1-\cos \alpha \varphi_{n j}\right)\left(\rho_{n}+\rho_{j}\right)\right]
\end{aligned}
$$

This completes the formulation of the model for multidirectional pedestrian streams. The number of equations should be the same as the number of streams being studied. The model can also be reduced to a one-dimensional Drake model when all $\varphi_{\mathrm{ij}}=0$.

\subsection{Model Calibration Method}

Although the data collected from an actual site can closely reflect reality, they cannot cover the full range of parameters as we cannot control the pedestrians to perform in situations with different intersecting angles and different levels of density. However, we have a set of useful data which was collected in a previous controlled experiment (Wong, et al., 2010). The experiments for the intersecting angles of 0,45 , 90, 135 and 180 degrees were performed with different levels of density. Therefore, the data collected from the controlled experiment sufficiently cover the full range of the parameters. However, an experiment is still not reality. To utilize the information that fully covers the range of parameters and the data from the field, we adopt Bayesian inference to perform the model calibration. In this way, the experimental data can provide an informative prior distribution and the field data can be used to update the coefficients. Hence, this enables us to finally obtain a model that is based on reliable experimental data and is updated by new evidence from a real site.

Bayesian inference is a method of statistical inference that uses Bayes' theorem to calculate how the prior distribution changes according to new evidence. It is a modeling approach for parameter estimation that integrates prior information and current information. The ultimate aim of Bayesian inference is to obtain the posterior distribution of all unknowns, i.e., the parameters of interest. The Bayesian modeling was performed using WINBUGS (Spiegelhalter et al., 2003), a flexible software program for the Bayesian analyses of complex statistical models using Markov chain Monte Carlo (MCMC) methods.

To perform a Bayesian analysis, the prior data distribution must be specified. Although the conflicting effects in multidirectional pedestrian flows are represented as the accumulation of the effects in each pair of conflicting streams, it is reasonable to adopt the calibrated parameters and their distributions used in a similar bidirectional model proposed by Xie et al. (2013). Xie et al. (2013) reported two sets of calibrated parameters. One set is calibrated with the data collected from the controlled experiment conducted by 
Wong et al. (2010). The other is calibrated with data collected from a real site (a crossing in Central, Hong Kong), but uses the previous experimental results as the prior distribution. Therefore, both these sets of calibrated parameters and their corresponding distributions will be separately used as the prior distributions for the calibration of our multidirectional model. However, the prior distribution of the freeflow speed, $\mathrm{V}_{\mathrm{f}}$, will be set as non-informative, i.e. with a very large standard deviation, as it depends on the type of walking facility.

To summarize, the multidirectional pedestrian stream model proposed in this study will be calibrated with the data collected from the observational survey in Mong Kok, which in turn are based on the prior distributions obtained from the controlled experiment and the field survey in Central, respectively. The exact prior distributions used are listed in Table 3 below:

[Insert Table 3 Here]

Finally, we used the deviance information criterion (DIC) and the posterior p-value as indices to evaluate the goodness-of-fit and to check the performance of the models. The DIC is useful in Bayesian model selection as it measures how well the model fits and considers the penalties on the number of parameters. Generally, the model with the lowest DIC value is preferred (Spiegelhalter et al., 2002).The posterior pvalue compares the predicted data from the model with the observed data. With the Bayesian framework, the predictive data can be simulated from the posterior predictive distribution. The goodness-of-fit can be evaluated by comparing the predictive data with the observed data. Assuming observations with the same parameters will be replicated in the future, another set of observed data can then be obtained. If the model is appropriate for the observed data, the replicated data should be very close to the observed data. Hence, the difference between the two sets of data will reveal the goodness-of-fit of the model. The posterior pvalue is defined as the probability that the replicated data are more extreme than the observed data. Therefore, the closer the posterior p-value is to 0.5 , the better the fit of the model (Gelman et al., 2004).

In addition to the Bayesian statistics, we adopted the mean absolute percentage error (MAPE), the root mean square error (RMSE) and the relative root mean square error (RRMSE) as statistics to evaluate the goodness-of-fit of the models.

\section{RESULTS AND DISCUSSION}

According to the model for multidirectional pedestrian flows proposed in section $3.1, \mathrm{n}=2$ for the controlled experiment and the field observation in Central, while $\mathrm{n}=4$ for the field observation in Mong Kok. Bayesian inference was adopted for the model calibration. For the parameters reflecting the interaction between pedestrians, $\theta, \beta$ and $\alpha$, the prior distributions were specified as in Table 3 . For the free-flow speed, which may depend on the condition of the walking facilities, the variance, $\sigma^{2}$, was set very large to provide enough flexibility in the prior distribution.

As shown in Table 4, the two groups of results are very similar. When the prior distribution is provided by the controlled experiment, the calibrated free-flow speed is $1.068 \mathrm{~m} / \mathrm{s}(95 \%$ BCIs: $1.061,1.075)$, the coefficient of isotropic effect $\theta$ is 0.063 (95\% BCIs: $0.060,0.067)$, the coefficient $\beta$ is 0.132 (95\% BCIs: $0.125,0.139)$ and the coefficient $\alpha$ is 1.227 (95\% BCIs: $1.168,1.297$ ). When the prior distribution is provided by the observation in Central, the calibrated free-flow speed is $1.071 \mathrm{~m} / \mathrm{s}(95 \%$ BCIs: 1.064 , 1.071), the coefficient of isotropic effect $\theta$ is 0.066 (95\% BCIs: $0.062 ., 0.070$ ), the coefficient $\beta$ is 0.132 (95\% BCIs: $0.125,0.138)$ and the coefficient $\alpha$ is 1.177 (95\% BCIs: $1.122,1.239)$.

[Insert Table 4 Here] 
Compared to the parameters originally calibrated from the controlled experiment and the observation in Central (Table 3), the values of the corresponding parameters are close, except for the coefficient $\beta$. The calibrated free-flow speed is similar to that in the controlled experiment, which is $1.074 \mathrm{~m} / \mathrm{s}$, indicating that the pedestrians in Mong Kok and the volunteers in the sports stadium walked at a similar leisurely pace. However, this is much lower than the calibrated free-flow speed observed in Central, which is 1.326 $\mathrm{m} / \mathrm{s}$ as reported in Xie et al. (2013). It is reasonable that the pedestrians at the crossing in Central walk faster than those walking leisurely in the shopping area in Mong Kok. The coefficients of the isotropic effect, $\theta$, for the two cases are also very close to the prior distributions (Table 3 ). It appears that the isotropic effect remains almost constant under the different pedestrian flow conditions. The coefficients of the angle, $\alpha$, are also similar to the prior values (Table 3), at between 1.177 to 1.3 , indicating that the conflicting effect induced by the angle is maximized when the angle is between $135^{\circ}$ and $150^{\circ}$. Finally, the coefficient of the conflicting effect, $\beta$, for the site in Mong Kok is 0.132 , which is much larger than the coefficients of 0.072 and 0.078 obtained in the controlled experiment and the observation in Central. As the exact form of the model changed from $n=2$ to $n=4$, the corresponding coefficient thus changed to represent the conflicting effect induced by the multiple conflicting streams.

To demonstrate the improvement in the accuracy of the models from one-directional to multidirectional pedestrian streams, we perform the same inference procedures using the same set of field data, although we disregard the stream directions and consider the flow as one stream of pedestrians. In this case, the proposed model reduces back to Drake's model and we only have the parameters $V_{\mathrm{f}}$ and $\theta$, the free-speed and the coefficient of isotropic effect. The calibration results are shown in Table 5 below:

\section{[Insert Table 5 Here]}

As shown in Table 5, the accuracy of the prediction is much lower than that of the multidirectional model. The DIC value for the univariate case is not comparable to that of the multivariate flow as the definitions of the dependent variables are different. Although the posterior p-values for both cases are very close to 0.5 , the values of the mean absolute percentage error (30.0\%) and relative root mean square error $(38.0 \%)$ are much larger than the corresponding values for the proposed multidirectional pedestrian stream model $(14.4 \%$ and $14.3 \%)$. This may provide strong evidence that the proposed multidirectional pedestrian stream model is superior to the previous bidirectional and one-dimensional models.

As we perform the Bayesian inference using WinBUGS, in which $\mathrm{V}_{1}, \mathrm{~V}_{2} \ldots \mathrm{V}_{\mathrm{n}}$ are assumed to have independent distributions, we need to test the validity of the assumption as the variables are dependent on each other according to the form of the model. To test whether the distributions of the speeds are independent of each other, we perform an analysis on the errors between the observed speeds and the predicted speeds computed from the proposed model with the calibrated coefficients. Let

$$
\varepsilon_{\mathrm{i}}=\mathrm{V}_{\mathrm{i}}^{\mathrm{pred}}-\mathrm{V}_{\mathrm{i}}^{\mathrm{obs}}
$$

where

$\mathrm{V}_{\mathrm{i}}^{\text {pred }}$ is the predicted speed of the the $\mathrm{i}^{\text {th }}$ stream;

$\mathrm{V}_{\mathrm{i}}{ }^{\text {obs }}$ is the predicted speed of the the $\mathrm{i}^{\text {th }}$ stream;

$\varepsilon_{\mathrm{i}}$ is error between the predicted speed and the observed speed of the $\mathrm{i}^{\text {th }}$ stream.

The study site comprises four streams of pedestrians with speeds of $V_{1}, V_{2}, V_{3}$ and $V_{4}$. Hence, the errors between the observed and estimated values are labeled as $\varepsilon_{1}, \varepsilon_{2}, \varepsilon_{3}$ and $\varepsilon_{4}$. The correlation between $\varepsilon_{1}, \varepsilon_{2}$, $\varepsilon_{3}$ and $\varepsilon_{4}$ is shown in Table 6 below:

[Insert Table 6 Here] 
As shown in Table 6, the correlation between any pair of error terms is very weak, which may indicate that it is still appropriate to assume that the error distributions of the speeds are independent.

In the multidirectional pedestrian model, there are countless combinations for the distribution of pedestrians in each stream and the intersecting angles between each set of two conflicting streams. To present some design charts to illustrate the relationship between speed and density, the directions of each of the four streams observed in the field survey are adopted as the fix values of the intersecting angles, i.e. the intersecting angles between the streams are set at $90^{\circ}$ for the adjacent streams and $180^{\circ}$ for the opposing streams. To plot the speed and density on a two-dimensional chart, the densities of Streams 2, 3 and 4 are set as equal so that they can be grouped together. Let

and

$$
\begin{gathered}
\rho_{234}=\rho_{2}+\rho_{3}+\rho_{4} \\
\rho_{2}=\rho_{3}=\rho_{4}
\end{gathered}
$$

Finally, the design charts for the speeds of Stream 1, Stream 2 (the same as Stream 4 as with the same density) and Stream 3 are plotted in Figure 3:

\section{[Insert Figure 3 Here]}

Figure 3 shows that the speed of each stream reduces as the density of Stream 1 increases from 1 to 6 $\mathrm{ped} / \mathrm{m}^{2}$, while the densities of the other streams remain constant. Figure 3(a) shows how $\mathrm{V}_{1}$ decreases as $\rho_{1}$ increases when $\rho_{234}$ is constant. When $\rho_{234}$ is relatively low $\left(\rho_{234}<1\right), V_{1}$ first decreases very slowly from the free-flow speed, as the system is nearly empty at this stage. However, when $\rho_{234}$ is larger $\left(\rho_{234}>=1\right), V_{1}$ drops quickly at the very beginning, as the presence of pedestrians in the other streams cause interactions that have a conflicting effect on Stream 1.

In addition, Figures 3(b) and (c) show the corresponding speeds for Streams 2, 3 and 4. Except when $\rho_{234}=0$, the speed drops sharply at the beginning of the curve, as the three streams interact with each other before the arrival of pedestrians in Stream 1. Therefore, once one pedestrian enters Stream 1, the increase in density in Stream 1 adds further conflicting effects to the whole system so that the speed of any of the three other streams will drop even faster.

Based on the design charts for speed/density depicted in Figure 3, the flow/density diagram can be plotted, as shown in Figure 4. As the flow is plotted against the total density for $\rho_{1}=\rho_{234}$, i.e. $\rho_{1}=\rho_{2}+\rho_{3}+\rho_{4}$, it is easy to compare this case with the bidirectional situations in Wong et al. (2010) and Xie et al. (2013).

\section{[Insert Figure 4 Here]}

As shown in Figure 4, the optimum total density of the pedestrian streams occurs at around $2.5 \mathrm{ped} / \mathrm{m}^{2}$, with the maximum flow being about $1.45 \mathrm{ped} / \mathrm{m} / \mathrm{s}$. As reported by Wong et al. (2010), under a balanced situation, the optimum total density for the bidirectional pedestrian streams is around $2.5 \mathrm{ped} / \mathrm{m}^{2}$, with the maximum flow in the controlled experiment being $1.6 \mathrm{ped} / \mathrm{m} / \mathrm{s}$. Considering that the average speed in the controlled experiment $(0.819 \mathrm{~m} / \mathrm{s})$ is lower than that in the field observation in Mong Kok $(0.96 \mathrm{~m} / \mathrm{s})$, it may be concluded that multiple conflicting streams from different directions will have a much larger impact on one stream than a single conflicting stream, even with the same density. A similar conclusion can also be drawn when these results are compared with those observed in Central, in which the optimum density is about $2 \sim 3 \mathrm{ped} / \mathrm{m}^{2}$ and the maximum flow is about $1.8 \sim 2.1 \mathrm{ped} / \mathrm{m} / \mathrm{s}$. This indicates that the pedestrians at the busy crossing walk at a much higher speed than those walking leisurely along the pedestrian street in Mong Kok. Figure 4 also shows the flows of the other three streams. Although the 
density of each of the three streams is only one third of that of Stream 1, the optimum flow of the streams is around $1.2 \mathrm{ped} / \mathrm{m} / \mathrm{s}$, which is slightly lower than that of Stream 1 . This indicates that the speed of the streams cannot be determined simply by the density.

\section{CONCLUSION}

Expanding on Drake's model, we developed a mathematical model to represent the movements of multidirectional pedestrian flows. In addition to stream density, we introduced the flow ratios and intersecting angles of the streams as attributes that influence the speed of the streams. A set of observational data (from the pedestrian street in Mong Kok) was collected for the model calibration. The movements of the pedestrians were video recorded and the corresponding macroscopic quantities were extracted to calibrate the model. Bayesian inference was adopted for the parameter calibration. The prior distribution for the Bayesian inference was provided by the calibration results reported by Xie et al. (2013), which are based on data collected from a controlled experiment performed by Wong et al. (2010) and an observational survey in Central conducted by Xie et al. (2013). The field data collected in Mong Kok was then used to update the model to reflect real-world situations of multidirectional pedestrian flows. These findings build on previous controlled experiments and observational studies on bidirectional pedestrian streams with oblique intersecting angles. The use of on-site observation helped us to understand the difference between the experimental and real situations and to improve the model. The results are generally consistent with those of previous studies. However, more observational surveys of different walking facilities should be conducted to make the model even more congruent with actual pedestrian behavior. This is an important area for future study.

\section{ACKNOWLEDGEMENTS}

The work described in this paper was supported by a Research Postgraduate Studentship and grants from the Research Grants Council of the Hong Kong Special Administrative Region, China (Project Nos. HKU7184/10E and HKU7175/12E).

\section{REFERENCES}

Abdelghany, A., Abdelghany, K., Mahmassani, H.S., \& Al-Gadhi, S.A. (2005). Microsimulation assignment model for multidirectional pedestrian movement in congested facilities. Bicycles and Pedestrians: Developing Countries, 123-132.

Ando, K., Ota, H., \& Oki., T. (1988). Forecasting the flow of people. Railway Research Review, 45(8), 814 (in Japanese).

Asano, M., Sumalee, A., Kuwahara, M., \& Tanaka, S. (2007). Dynamic Cell Transmission-Based Pedestrian Model with Multidirectional Flows and Strategic Route Choices. Transportation Research Record: Journal of the Transportation Research Board, 2039, 42-49.

Blue, V.J. \& Adler, J.L. (2000). Modeling four-directional pedestrian flows. Transportation Research Record, 1710: Traffic Flow Theory and Highway Capacity, 20-27.

Daamen, W. \& Hoogendoorn, S.P. (2003). Experimental research of pedestrian walking behavior. Transportation Research Record, 1828, 20-30.

Daamen, W., Hoogendoorn, S.P., \& Bovy, P.H.L. (2005). First-order pedestrian traffic flow theory. Traffic Flow Theory 2005, 43-52.

Drake, J., Schofer, J., \& May, A. (1967). A statistical analysis of speed density hypotheses. Proceedings of the Third International Symposium on the Theory of Traffic Flow, New York, NY, 112-117.

Gelman, A. (2004). Bayesian Data Analysis. Chapman \& Hall/CRC, Boca Raton, FL.

Hediyeh, H., Sayed, T., Zaki, M.H., \& Ismail, K. (2014a) Automated analysis of pedestrian crossing speed 
behavior at scramble-phase signalized intersections using computer vision techniques. International Journal of Sustainable Transportation, 8, 382-397.

Hediyeh, H., Sayed, T., Zaki, M.H., \& Mori, G. (2014b) Pedestrian gait analysis using automated computer vision techniques. Transportmetrica A: Transport Science, 10, 214-232.

Helbing, D., Buzna, L., Johansson, A., \& Werner, T. (2005). Self-organized pedestrian crowd dynamics: Experiments, simulations, and design solutions. Transportation Science, 39, 1-24.

Hoogendoorn, S.P. \& Daamen W. (2005). Pedestrian behavior at bottlenecks. Transportation Science, 39(2), 147-159.

Huang, L., Wong, S.C., Zhang, M.P., Shu, C.W., \& Lam, W.H.K. (2009). Revisiting Hughes' dynamic continuum model for pedestrian flow and the development of an efficient solution algorithm. Transportation Research Part B-Methodological, 43(1), 127-141.

Hughes, R.L. (2002). A continuum theory for the flow of pedestrians. Transportation Research Part BMethodological, 36(6), 507-535.

Jiang, Y.Q., Xiong, T., Wong, S.C., Shu, C.W., Zhang, M.P., Zhang, P., \& Lam, W.H.K. (2009). A reactive dynamic continuum user equilibrium model for bi-directional pedestrian flows. Acta Mathematica Scientia, 29(6), 1541-1555.

Jiang, Y.Q., Wong, S.C., Zhang, P., Liu, R.X., Duan, Y.L., \& Choi, K. (2012). Numerical simulation of a continuum model for bi-directional pedestrian flow. Applied Mathematics and Computation, 218(10), 6135-6143.

Kretz, T., Grunebohm, A., Kaufman, M., Mazur, F., \& Schreckenberg, M. (2006a). Experimental study of pedestrian counterflow in a corridor. Journal of Statistical Mechanics-Theory and Experiment, P10001.

Kretz, T., Grunebohm, A., \& Schreckenberg, M.. (2006b). Experimental study of pedestrian flow through a bottleneck. Journal of Statistical Mechanics-Theory and Experiment, P10014.

Lam, W.H.K. \& Cheung, C.Y. (2000). Pedestrian speed/flow relationships for walking facilities in Hong Kong. Journal of Transportation Engineering, ASCE, 126, 343-349.

Lam, W.H.K., Lee, J.Y.S., Chan, K.S., \& Goh, P.K. (2003). A generalised function for modeling bidirectional flow effects on indoor walkways in Hong Kong. Transportation Research Part A, 37, 789810.

Lam, W.H.K., Lee, J.Y.S., \& Cheung, C.Y. (2002). A study of the bi-directional pedestrian flow characteristics at Hong Kong signalized crosswalk facilities. Transportation, 29, 169-192.

Moussaid, M., Helbing, D., Garnier, S., Johansson, A., Combe, M., \& Theraulaz, G. (2009). Experimental study of the behavioural mechanisms underlying self-organization in human crowds. Proceedings of the Royal Society B-Biological Sciences, 276, 2755-2762.

Ntzoufras, I. (2009). Bayesian Modeling Using WinBUGS, Hoboken, N.J., Wiley.

Potts, R. B., \& Oliver, R. M. (1972). Flows in Transportation Networks: Academic Press.

Spiegelhalter, D.J., Best, N.G., Carlin, B.P., \& van der Linde, A. (2002). Bayesian measures of model complexity and fit. Journal of the Royal Statistical Society. Series B (Statistical Methodology), 64(4), 583-639.

Wong, S.C., Leung, W.L., Chan, S.H., Lam, W.H.K., Yung, N.H.C., Liu, C.Y., \& Zhang, P. (2010). Bidirectional pedestrian stream model with oblique intersecting angle. Journal of Transportation Engineering-ASCE 136, 234-242.

Xia, Y.H., Wong, S.C., \& Shu, C.W. (2009). Dynamic continuum pedestrian flow model with memory effect. Physical Review E, 79(6).

Xie, S. Q., Wong, S. C., Lam, W. H. K., \& Chen, A. (2013). Development of a Bidirectional Pedestrian Stream Model with an Oblique Intersecting Angle. Journal of Transportation Engineering, 139(7), 678-685.

Xiong, T., Zhang, M., Shu, C.W., Wong, S.C ., \& Zhang, P. (2011). High-order computational scheme for a dynamic continuum model for bi-directional pedestrian flows. Computer-Aided Civil and Infrastructure Engineering, 26(4), 298-310.

Zhang, J. \& Seyfried, A. (2014). Comparison of intersecting pedestrian flows based on experiments. Physica A: Statistical Mechanics and its Applications, 405(0), 316-325. 
Tables

Table 1 Summary of the pedestrian flows

\begin{tabular}{lc}
\hline Sample Size & 18,465 \\
Average Speed $(\mathrm{m} / \mathrm{s})$ & 0.96 \\
Standard Deviation of Speed $(\mathrm{m} / \mathrm{s})$ & 0.56 \\
Average Density $\left(\mathrm{ped} / \mathrm{m}^{2}\right)$ & 0.65 \\
Standard Deviation of Density $\left(\mathrm{ped} / \mathrm{m}^{2}\right)$ & 0.32 \\
\hline
\end{tabular}

Table 2 Average speed and density of the four pedestrian streams

\begin{tabular}{ccc}
\hline Stream & Average Speed $(\mathrm{m} / \mathrm{s})$ & $\begin{array}{c}\text { Average Density } \\
\left(\mathrm{ped} / \mathrm{m}^{2}\right)\end{array}$ \\
\hline Stream 1 $(\mathrm{S} \rightarrow \mathrm{N})$ & 0.93 & 0.222 \\
Stream 2 $(\mathrm{E} \rightarrow \mathrm{W})$ & 0.92 & 0.079 \\
Stream 3 $(\mathrm{N} \rightarrow \mathrm{S})$ & 0.96 & 0.263 \\
Stream 4 $(\mathrm{W} \rightarrow \mathrm{E})$ & 1.00 & 0.084 \\
\hline
\end{tabular}

Table 3 Prior distributions from the controlled experiment and the observation in Central

\begin{tabular}{c|c|c|c|c}
\hline \multirow{2}{*}{ Parameters } & \multicolumn{2}{|c|}{ Controlled Experiment } & \multicolumn{2}{c}{ Field Observation in Central } \\
\cline { 2 - 5 } & Mean & $\begin{array}{c}\text { Standard } \\
\text { Deviation }\end{array}$ & Mean & $\begin{array}{c}\text { Standard } \\
\text { Deviation }\end{array}$ \\
\hline$\theta$ & 0.062 & $2.18 \times 10^{-03}$ & 0.065 & $2.02 \times 10^{-03}$ \\
\hline$\beta$ & 0.072 & $4.27 \times 10^{-03}$ & 0.078 & $4.06 \times 10^{-03}$ \\
\hline$\alpha$ & 1.271 & $3.2 \times 10^{-02}$ & 1.214 & $3.2 \times 10^{-02}$ \\
\hline
\end{tabular}

Table 4 Model calibration results

\begin{tabular}{|c|c|c|c|c|c|c|c|}
\hline \multicolumn{2}{|c|}{ Prior distribution } & \multicolumn{3}{|c|}{ Controlled Experiment } & \multicolumn{3}{|c|}{ Observation in Central } \\
\hline & & Estimate & & BCIs) & Estin & & BCIs) \\
\hline \multirow{4}{*}{$\begin{array}{l}\text { Calibrated } \\
\text { Parameters }\end{array}$} & $\mathrm{V}_{\mathrm{f}}$ & 1.068 & 1.061 & 1.075 & 1.071 & 1.064 & 1.071 \\
\hline & $\theta$ & 0.063 & 0.060 & 0.067 & 0.066 & 0.062 & 0.070 \\
\hline & $\beta$ & 0.132 & 0.125 & 0.139 & 0.132 & 0.125 & 0.138 \\
\hline & $\alpha$ & 1.227 & 1.168 & 1.297 & 1.177 & 1.122 & 1.239 \\
\hline \multicolumn{2}{|l|}{$\begin{array}{c}\text { Posterior } \\
\text { p-value }\end{array}$} & \multicolumn{3}{|c|}{0.5065} & \\
\hline \multicolumn{2}{|l|}{ DIC } & \multicolumn{3}{|c|}{72236.7} & \multicolumn{3}{|c|}{72237.1} \\
\hline \multicolumn{2}{|l|}{ MAPE } & \multicolumn{3}{|c|}{$14.4 \%$} & \multicolumn{3}{|c|}{$14.3 \%$} \\
\hline \multicolumn{2}{|l|}{$\begin{array}{c}\text { RMSE } \\
\text { RRMSF }\end{array}$} & \multicolumn{3}{|c|}{0.297} & \multicolumn{3}{|c|}{0.284} \\
\hline \multicolumn{2}{|l|}{ RRMSE } & \multicolumn{3}{|c|}{$30.2 \%$} & \multicolumn{3}{|c|}{$28.9 \%$} \\
\hline
\end{tabular}


Table 5 Model calibration results for univariate flow

\begin{tabular}{|c|c|c|c|c|c|c|c|}
\hline \multicolumn{2}{|c|}{ Prior distribution } & \multicolumn{3}{|c|}{ Controlled Experiment } & \multicolumn{3}{|c|}{ Observation in Central } \\
\hline & & \multirow{2}{*}{$\begin{array}{l}\text { Estimate } \\
1.006\end{array}$} & \multicolumn{2}{|c|}{ (95\% BCIs) } & \multirow{2}{*}{$\begin{array}{c}\text { Estimate } \\
1.007\end{array}$} & \multicolumn{2}{|c|}{ (95\% BCIs) } \\
\hline Calibrated & $V_{f}$ & & 1.000 & 1.012 & & 1.001 & 1.012 \\
\hline Parameters & $\theta$ & 0.072 & 0.068 & 0.076 & 0.074 & 0.070 & 0.078 \\
\hline $\begin{array}{l}\text { Posterior } \\
\text { p-value }\end{array}$ & & & 0.4967 & & & 0.4967 & \\
\hline DIC & & & 15488.8 & & & 15482.1 & \\
\hline MAPE & & & $30.0 \%$ & & & $30.0 \%$ & \\
\hline RMSE & & & 0.368 & & & 0.368 & \\
\hline RRMSE & & & $38.0 \%$ & & & $38.0 \%$ & \\
\hline
\end{tabular}

Table 6 Correlation between error terms, $\varepsilon_{1}, \varepsilon_{2}, \varepsilon_{3}$ and $\varepsilon_{4}$

\begin{tabular}{lllll}
\hline & $\varepsilon_{1}$ & $\varepsilon_{2}$ & $\varepsilon_{3}$ & $\varepsilon_{4}$ \\
\hline$\varepsilon_{1}$ & 1 & & & \\
$\varepsilon_{2}$ & 0.031795 & 1 & & \\
$\varepsilon_{3}$ & 0.043973 & 0.007749 & 1 & \\
$\varepsilon_{4}$ & 0.010478 & 0.027278 & 0.0356 & 1 \\
\hline
\end{tabular}




\section{List of Figure Captions}

Figure 1 Pedestrian Street in Mong Kok

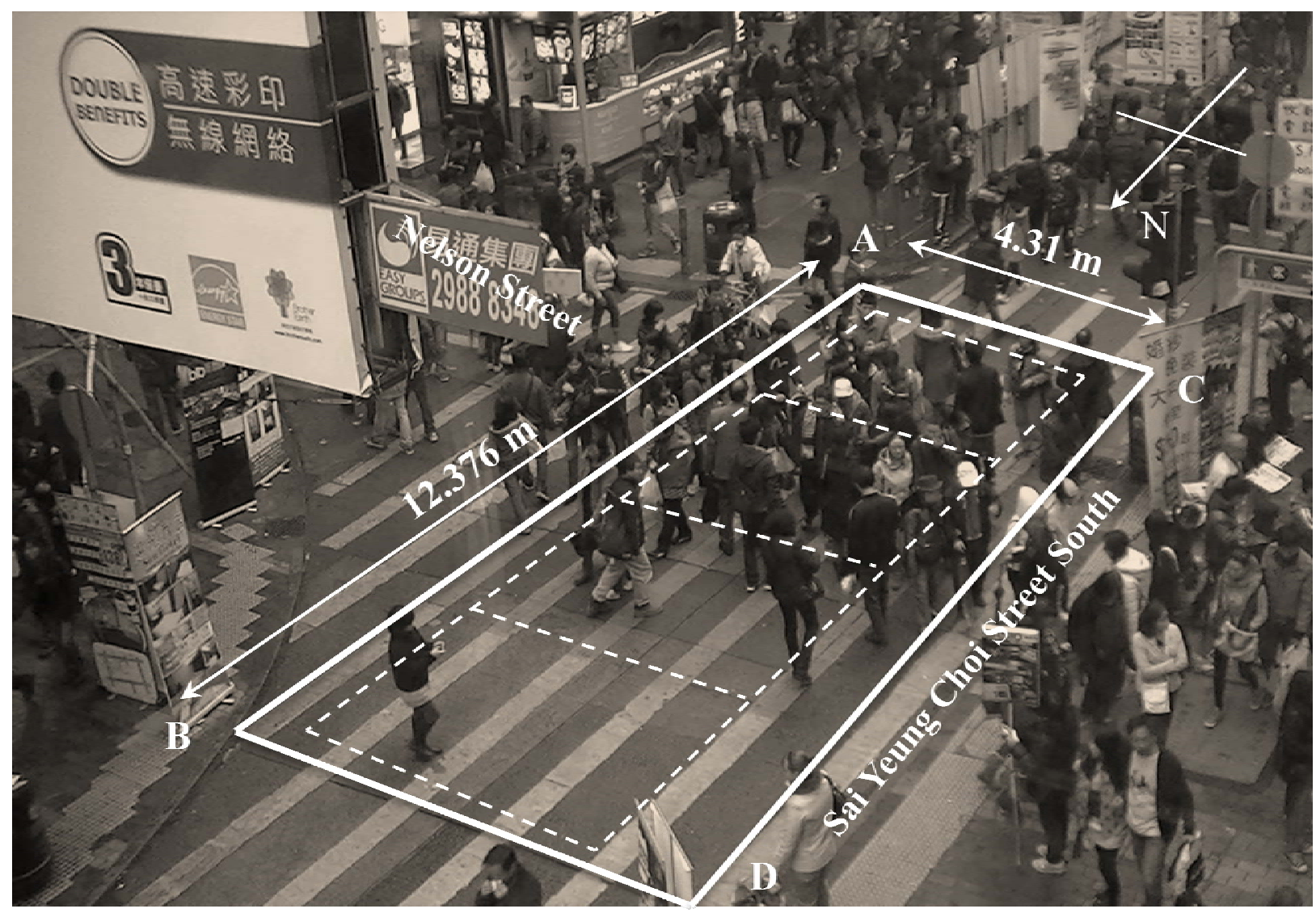


Figure 2 Classification of Directions

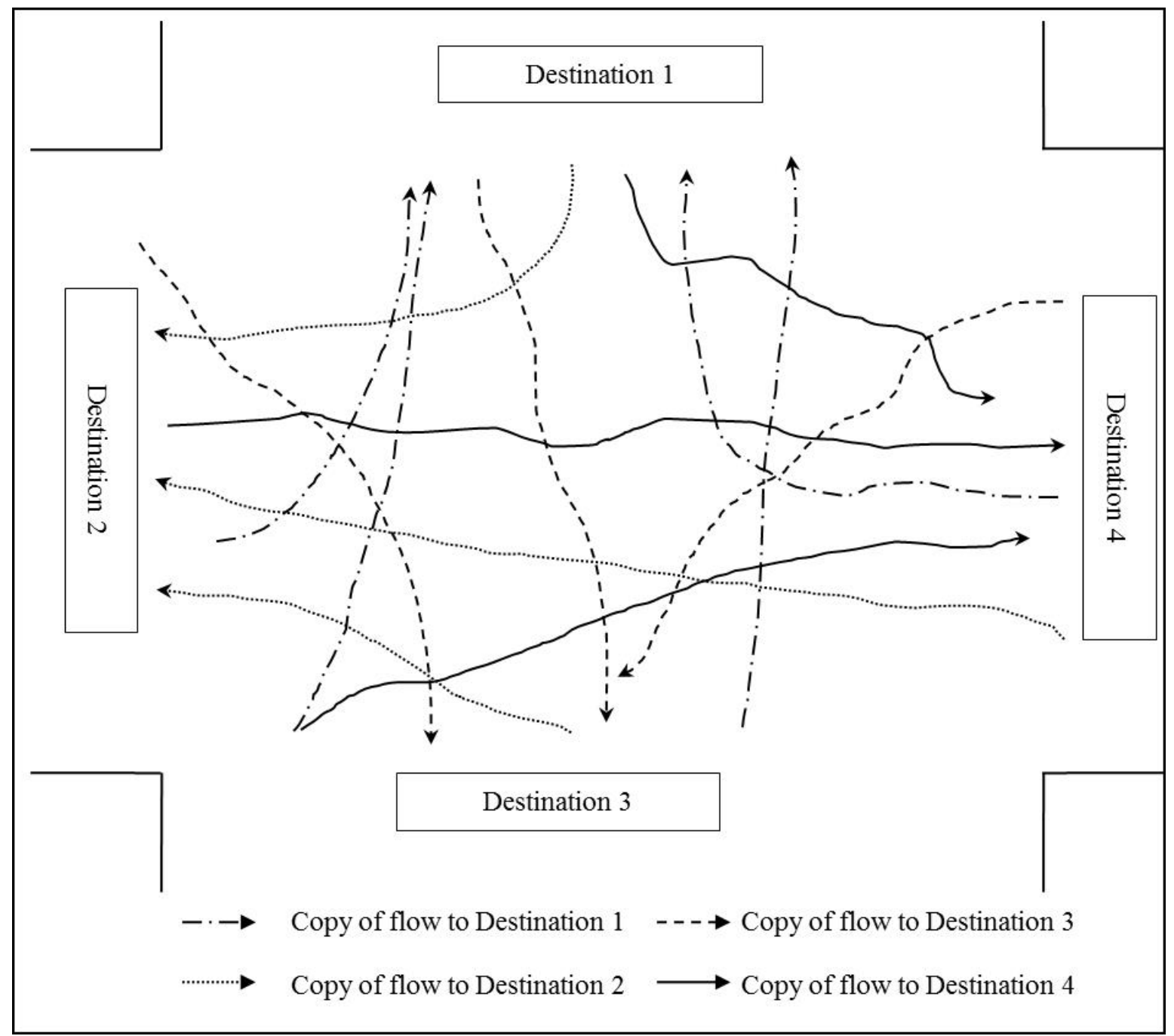


Figure 3 Design Charts for the Speed of the Streams

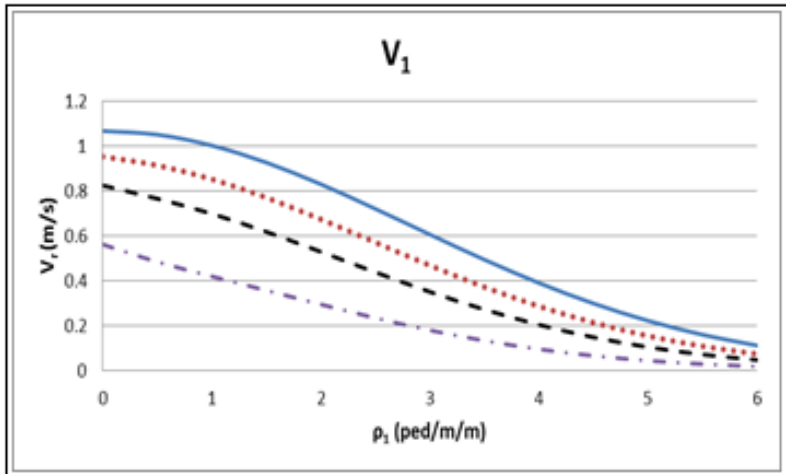

(a)

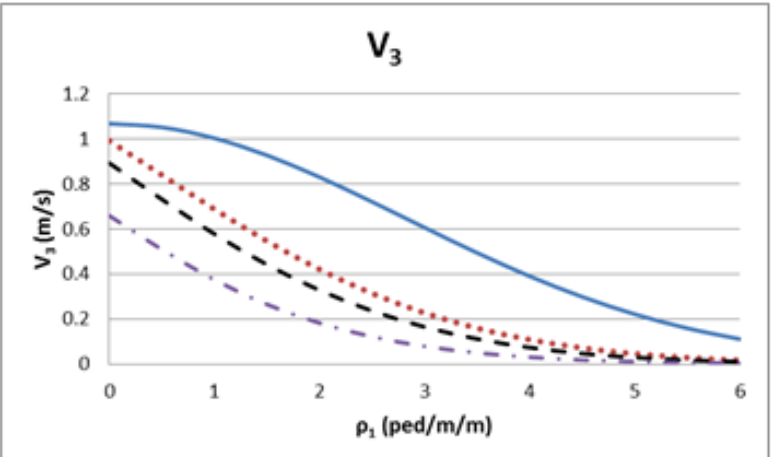

(c)

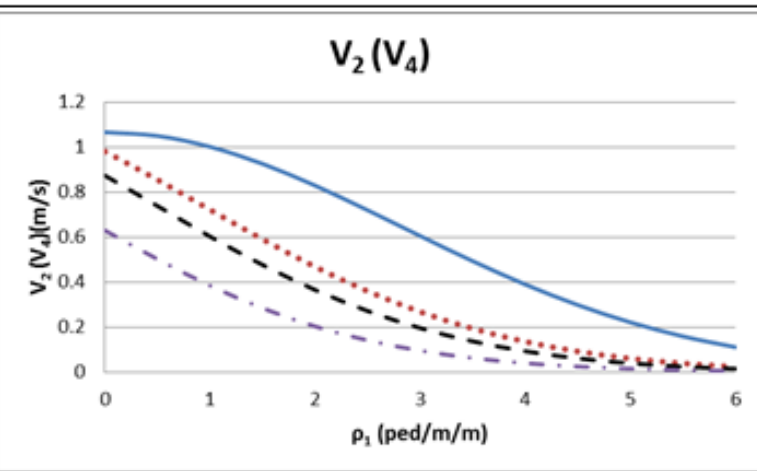

(b)

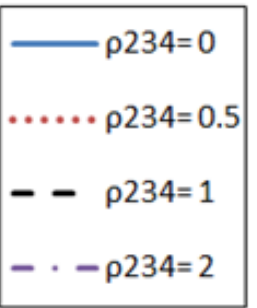

Figure 4 Flow-total Density Relationship $\left(\rho_{1}=\rho_{2}+\rho_{3}+\rho_{4}\right)$

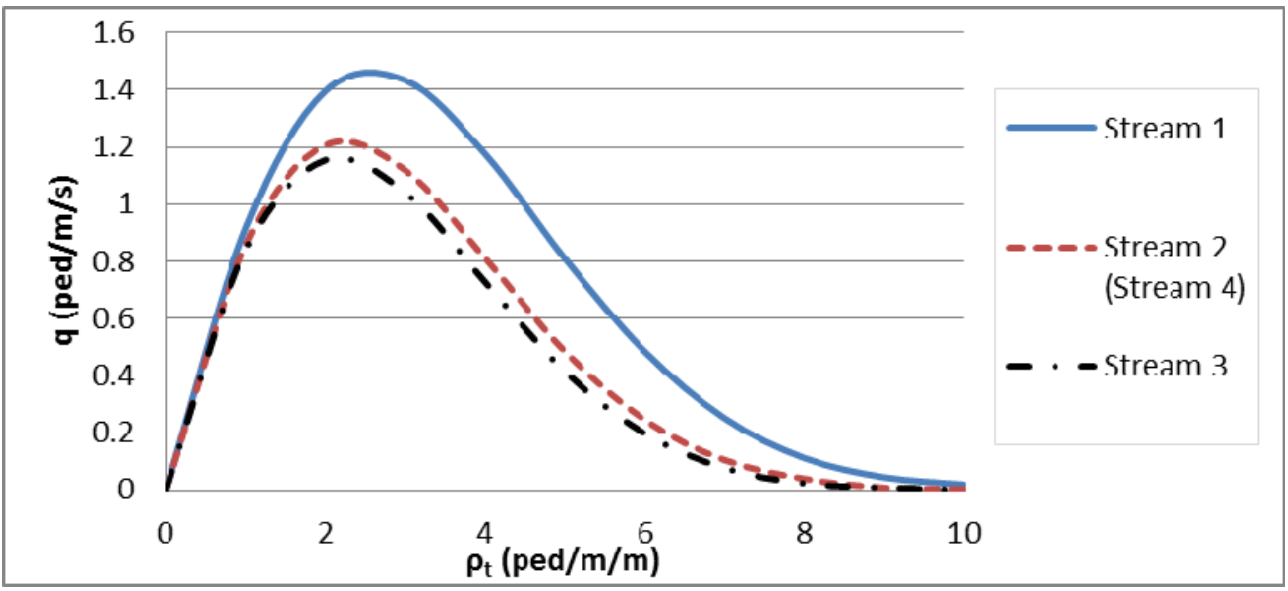

